\title{
Cognitive Impairment in Children with Adenotonsillar Hypertrophy
}

Mohammed A. Gomaa ${ }^{*}$, Haytham Mamdoh', Zienab Khalaf', Wafaa H Abdel Hakeem², Effat A. Zaky².
${ }^{1}$ Department of Otolaryngology, Minia faculty of Medicine at Minia University, Minia, Egypt.
${ }^{2}$ Department of Otolaryngology, Phoniatrics unite, Minia faculty of Medicine at Minia University, Minia, Egypt.

*Corresponding Author: Mohammed A. Gomaa, Department of Otolaryngology, Minia faculty of Medicine at Minia University, Minia, Egypt.

Received date: November 11, 2020; Accepted date: December 21, 2020; Published date: January 01 , 2021

Citation: Gomaa M A, Mamdouh H, khalaf Z, Abd El-hakeem WH, Zaky EA. (2021) Cognitive Impairment in Children with Adenotonsillar Hypertrophy. J. Neuroscience and Neurological Surgery. 8(1); DOI:10.31579/2578-8868/147

Copyright: (c) 2021 Mohammesd A. Gomaa, This is an open-access article distributed under the terms of The Creative Commons Attribution License, which permits unrestricted use, distribution, and reproduction in any medium, provided the original author and source are credited

\begin{abstract}
A prospective case control study
\section{Abstract:}

Pediatric OSA affects 1 to $3 \%$ of the population and appears to affect boys and girls equally [4]. The most commonly cause of pediatric OSA is adenotonsillar hypertrophy. Thus, the primary treatment is adenotonsillectomy. Pediatric OSA has been associated with some psychological problems, of which neurocognitive and depression, difficulties particularly in memory, attention, learning and executive function, are the most widely reported. The neurocognitive deficits is due to the adverse effects of sleep fragmentation and/or intermittent hypoxia .Scholastic performance have been reported in little studies of pediatric OSA, and such findings may underscore more extensive behavioral disturbances such as restlessness, aggressive behavior, excessive daytime sleepiness and poor test performances. The aim of this study was to evaluate the effect of adenotonsillectomy (AT), in children with Obstructive Sleep Apnea (OSA), on the cognitive and scholastic achievement.
\end{abstract}

Keywords: obstructive sleep apnea; adenotonsillectomy; cognition.

\section{Introduction:}

Obstructive sleep apnea (OSA) in children is a breathing disorder that results in repeated and/or prolonged airway obstructions during sleep, in the form of apneas and hypopneas throughout the night [1]. The highest incidence of pediatric OSA occurs in early childhood [2], particularly between 2 and 8 years of age [3]. Pediatric OSA affects 1 to $3 \%$ of the population and appears to affect boys and girls equally [4]. The most [5] commonly cause of pediatric OSA is adenotonsillar hypertrophy. Thus, the primary treatment is adenotonsillectomy, which accounts for more than 500,000 procedures annually in the United States alone [5]. OSA is known as sleep-disordered breathing [6] (SDB). This spectrum ranges in severity from habitual snoring to upper airway resistance syndrome (UARS) to OSA. As OSA influence about one to three percent of pediatric population [6], it has been associated with some psychological problems, of which neurocognitive and depression, difficulties particularly in memory, attention, learning and executive function, are the most widely reported. The neurocognitive deficits is due to the adverse effects of sleep fragmentation and/or intermittent hypoxia [7]. Some studies have reported the presence of significantly reduced IQ scores compared with control children [8]. Scholastic performance have been reported in little studies of pediatric OSA, and such findings may underscore more extensive behavioral disturbances such as restlessness, aggressive behavior, excessive daytime sleepiness and poor test performances [9]. While Marcus et al. [10] pointed that the surgical treatment of pediatric OSA didn't did not significantly improve attention or executive function as measured by neuropsychological testing but did reduce symptoms and improve secondary outcomes of behavior, quality of life, and polysomnographic findings, thus providing clues of beneficial effect of early AT.

The purpose of the present study is to evaluate the effect of adenotonsillectomy (AT) on the scholastic and cognitive achievement of children with OSA in order to establish a plan for proper management of those children.

\section{Materials and Methods}

The current study is a prospective one that done at the Department of Otorhinolaryngology from October 2016 to July 2019. It was approved by the Research ethics committee of the Faculty of Medicine, Minia University and signed informed consent was obtained from parents/ guardian of the children. One hundred Fifty children suffering from recurrent attacks of tonsillitis with or without chronic nasal obstruction were selected as pool for the present study. They were 73 males $(48 \%)$ and 77 females (52\%), with a mean age $101.5 \pm 15.6$ of and a range of (72120) months.

\section{Methods:}

Inclusion criteria were as follows: (1) Children with age ranging (5-12) years. (2) Well diagnosed with obstructive sleep apnea (OSA) due to adenotonsillar hypertrophy.

The exclusion criteria were as follows: (1) Age below 5 years and above 12 years; (2) Children taking sleep (hypnotics) or neurobehavioral drugs; (3) Children with Previous adenotonsillectomy; (4) Children with craniofacial syndromes; (5) Children with hearing impairment (SNHL); (6) Obese children (Body Mass Index (BMI) $\geq 30$ ). 


\section{All one hundred fifty children were subjected to the} following protocol to classify the study and control groups

1-Personal history were obtained from parents/ Guardian of the children, with special attention to sleep history including snoring.

2-Auditory perceptual assessment (APA) of speech (degree of closed nasality). The speech of each case were assessed from two expert phoniatricians for the degree of closed nasality (From I to IV) after recording of the patient's speech for a nasal sentence [11].

3- (ENT) Examination Otolaryngological examination: Oral and oropharyngeal examination (lips, teeth, tongue, hard and soft palates, uvula, tonsillar pillars, tonsils, lateral and posterior pharyngeal walls) was examined by Otolaryngologist with a tongue blade and a good light. Then tonsillar hypertrophied were graded by Brodsky scale (From zero to 4) [5].

Examination of the nasopharynx through nasopharyngoscopy, for evaluation of the velopharyngeal area (velar mobility, lateral pharyngeal wall mobility, presence of adenoid and degree of narrowing the air column, type of closure, presence or absence of passivent's ridge).

4- Polysomonography PSG was performed using a digital polygraph system (Grass-Telefactor Twin version 2.6; Astro-Med Inc., West Warwick, RI, USA). The data were manually scored according to the American Academy of Sleep Medicine , Version 2.0 Berry et al., [11].polysomnography (PSG) study using standard clinical pediatric techniques and a commercially available sleep system for diagnosis of OSA. The current gold standard for the diagnosis of OSA, as recommended by the American Academy of Pediatrics (AAP), is a PSG study. Polysomnographic diagnostic criteria for OSA depends on the following parameters Apnea-Hypoapnea index (AHI) (represents the average number of obstructive or partially obstructive events per hour of sleep and considered abnormal if greater than 1 in children), a minimum oxygen saturation (less than 92 percent (average: 96 percent \pm 2 percent) is considered abnormal in children). According to the inclusion criteria in table-2 we classified the patient to study group and control group [1].

We selected one hundred patients according to inclusion and exclusion criterias which were mentioned before, fifty child as study group with OSA that documented by PSG and fifty child suffer from chronic tonsillitis without OSA as a control group, . Both groups were subjected to protocol of evaluation the psycholinguistic abilities and IQ preoperatively as follow:

1-Parents interview and history including complaint, personal data, personal history, in addition to the clinical findings of prolonged upper airway obstructive symptoms, including OSA (recurrent episodes of apnea and hypopnea, secondary to collapse of the upper airways during sleep), snoring, mouth breathing, adenoid fancies, enuresis, and restlessness during sleep (if accompanied by snoring at night). History of arousal or awakening, and distrust sleep continuity.

2-Auditory perceptual assessment of speech (degree of closed nasality). The speech of each case were assessed from two experts for the degree of closed nasality after recording of the patient's speech for a nasal sentence [11].

3-Audiological evaluation included middle ear assessment through immitancemetry (Tympanometry and Acoustic Reflex threshold recording) and hearing assessment. According to the age of child. Hearing assessment was performed through one of the following methods: Free field Audiometry and Behavioral Observational Audiomety (BOA).pure tone audiometry (Conditioned play or conventional audiometry).
4-Psychometric evaluation by Intelligence Quotient "IQ" using Stanford Binet Intelligence Scale (4th edition). The Stanford-Binet scale tests intelligence across four areas: verbal reasoning, quantitative reasoning, abstract /visual reasoning, and short-term memory. The areas are covered by 15 subtests. Raw scores were based on the number of items answered, and were converted into a standard age score corresponding to age group. Mental age was determined for each child: $I . Q=$ Mental age chronological age $\times 100$ Gary [13].

5-Illinois test of psycholinguistic abilities (ITPA):

It is an individually administered battery of tests designed to measure the spoken and written language abilities of children aged 5-12 it may be used for determining specific strengths and weakness among language abilities, for early identification of children at-risk for school failure.

It consists of twelve subtests Adel Aziz A et al., [14].

6-Percentage of the child's scores of his subjects (Academic performance) in two academic terms, which were obtained from academic reports taken from the school.

All patients (cases and control groups) underwent Adenotonsillectomy (AT) under general anesthesia. Then finally after eight months of adenotonsillectomy all the patients were followed by:

- APA of speech.

- IQ test and mental age.

- Tympanogram

- Illinois test.

- Academic performance of the child

The data was collected from every battery of evaluation and subjected to I.B.M. compatible computer using software SPSS (Statistical Package for social science) for windows version 13 for statistical evaluation. Graphics were done by Excel. Quantitative data were presented by mean and standard deviation, while qualitative data were presented by frequency distribution. Wilcoxon signed-rank test for ordinal qualitative data between the two times: Significant level at P-value <

0.05. Paired samples T-test for parametric quantitative data between the two times: Significant level at P-value $<0.05$. Pearson's correlation: Significant level at P-value $<0.05$. Spearman's rho correlation. Significant level at $\mathrm{P}$-value $<0.05 \mathrm{P}$-value was considered statistically significant (S) if $<0.05$ and highly significant (HS), if $<0.001$ and no significant (NS) if $>0.05$.

\section{Results}

The study group had age range between (7-10 years) and mean 8.8 \pm 14 .6. They were 29 males and 21 females.

All patients in case group suffer from Moderate to severe closed nasality according to auditory perceptual assessment, Grade III to IV with tonsillar hypertrophied as measured by Brodsky scale, positive signs of Adenoid faces, Symptoms of OSA, the adenoid occupied $50-100 \%$ of the choanal area. PSG of the study group revealed that Apnea-Hypopnea index $(\mathrm{AHI})>1$ and oxygen saturation less than 92 percent. The control group (children without OSA) included 50 children, they have mild closed nasality according to auditory perceptual assessment, Grade 0 to I of tonsillar hypertrophied with by Brodsky scale, negative signs of Adenoid faces, no Symptoms of OSA, the adenoid occupied less than 25 $\%$ choanal area and with the PSG the had Apnea-Hypopnea index (AHI) $<1$ and oxygen saturation more than $\geq 96$ percent. The age of control group between ( $7-9$ years) and mean $7.9 \pm 14$.6. They were 26 male and 24 female (table 1-2). 


\begin{tabular}{|c|c|c|c|c|}
\hline & & Case(50) & Control (50) & $\mathrm{P}$ value \\
\hline Age & $\begin{array}{l}\text { Range } \\
\text { Mean } \pm \text { SD }\end{array}$ & $\begin{array}{l}(7-10 \text { years }) \\
8.8 \pm 14.6\end{array}$ & $\begin{array}{l}(7-9 \text { years) } \\
7.9 \pm 14.6\end{array}$ & 0.1 \\
\hline Sex & $\begin{array}{l}\text { Male } \\
\text { Female }\end{array}$ & $\begin{array}{l}29(58 \%) \\
21(42 \%)\end{array}$ & $\begin{array}{l}26(52 \%) \\
24(48 \%)\end{array}$ & 0.3 \\
\hline
\end{tabular}

Non- significant ( $p>0.05)$, significant ( $p<0.05)$, -highly significant $(p<0.001)$.

Table 1: Distribution of age and sex of study and control group.

\begin{tabular}{|c|c|c|c|c|}
\hline & & Case (50) & Control (50) & $P$ value \\
\hline APA & $\begin{array}{l}\text { No Closed } \\
\text { Mild } \\
\text { Moderate } \\
\text { Severe }\end{array}$ & $\begin{array}{l}0(0 \%) \\
0(0 \%) \\
32(64 \%) \\
18(36 \%)\end{array}$ & $\begin{array}{l}23(46 \%) \\
27(54 \%) \\
0(0 \%) \\
0(0 \%)\end{array}$ & $<0.001^{*}$ \\
\hline $\begin{array}{l}\text { Brodsky grade of } \\
\text { tonsils }\end{array}$ & $\begin{array}{l}0 \\
\text { I } \\
\text { II } \\
\text { III } \\
\text { IV }\end{array}$ & $\begin{array}{l}0 \\
0 \\
0 \\
32 \\
18\end{array}$ & $\begin{array}{l}28 \\
22 \\
0 \\
0 \\
0\end{array}$ & $<0.001 *$ \\
\hline Adenoid faces & $\begin{array}{l}\text { Positive } \\
\text { negative }\end{array}$ & $\begin{array}{l}50 \\
0\end{array}$ & $\begin{array}{l}0 \\
50\end{array}$ & $<0.001 *$ \\
\hline $\begin{array}{l}\text { Endoscopic grading of } \\
\text { adenoid. }\end{array}$ & $\begin{array}{l}\text { occupied more than } 50- \\
100 \% \text { choanal area } \\
\text { occupied less than } 25 \% \\
\text { of the choanal area }\end{array}$ & $\begin{array}{l}50 \\
0\end{array}$ & $\begin{array}{l}0 \\
50\end{array}$ & $<0.001 *$ \\
\hline PSG:AHI & $\begin{array}{l}>1 \\
<1\end{array}$ & $\begin{array}{l}50 \\
0\end{array}$ & $\begin{array}{l}0 \\
50\end{array}$ & $<0.001^{*}$ \\
\hline $\begin{array}{l}\text { PSG:O2 } \\
\text { saturation }\end{array}$ & $\begin{array}{l}<92 \\
\geq 96\end{array}$ & $\begin{array}{l}50 \\
0\end{array}$ & $\begin{array}{l}0 \\
50\end{array}$ & $<0.001 *$ \\
\hline
\end{tabular}

Non- significant ( $p>0.05)$, significant $(p<0.05)$, -highly significant $(p<0.001)$.

Table 2: Distribution of both group according to APA, Brodsky grade Tonsils enlargement and adenoid faces and PSG.

Preoperative assessment of both groups regarding psycholinguistic abilities, Academic performance, and middle ear function. The results revealed a highly statistically significant difference were obtained between the two groups as study group showed marked closed nasality (table-3), also symptoms of OSA were more apparent in the study group compared to control group (table-4).

\begin{tabular}{|c|l|l|l|l|}
\hline \multirow{2}{*}{ APA } & Case & control & \multirow{2}{*}{ P value } \\
\cline { 3 - 4 } & & $\mathrm{N}=50$ & $\mathrm{~N}=50$ & \\
\hline \multirow{5}{*}{ Preoperative } & No Closed & $0(0 \%)$ & $23(46 \%)$ & \\
& Mild & $0(0 \%)$ & $27(54 \%)$ & \multirow{2}{*}{$<0.001^{*}$} \\
& Moderate & $32 \mathrm{~s}(64 \%)$ & $0(0 \%)$ & \\
& Severe & $18(36 \%)$ & $0(0 \%)$ & \\
\hline \multirow{5}{*}{ Postoperative } & No Closed & $22(44 \%)$ & $23(46 \%)$ & \\
& Mild & $26(52 \%)$ & $27(54 \%)$ & 0.08 \\
& Moderate & $2(4 \%)$ & $0(0 \%)$ & \\
& Severe & $0(0 \%)$ & $0(0 \%)$ & \\
\hline
\end{tabular}

Non- significant $(p>0.05)$, significant $(p<0.05)$, -highly significant $(p<0.001)$. 


\begin{tabular}{|c|c|c|c|c|}
\hline \multirow[t]{2}{*}{ Symptoms of OSA } & & Case & control & \multirow{2}{*}{$P$ value } \\
\hline & & $\mathrm{N}=50$ & $\mathrm{~N}=50$ & \\
\hline Snoring & $\begin{array}{l}\text { Yes } \\
\text { No }\end{array}$ & $\begin{array}{l}50(100 \%) \\
0(0 \%)\end{array}$ & $\begin{array}{l}5(10 \%) \\
45(90 \%)\end{array}$ & $<0.001 *$ \\
\hline Mouth Breathing & $\begin{array}{l}\text { Yes } \\
\text { No }\end{array}$ & $\begin{array}{l}50(100 \%) \\
0(0 \%)\end{array}$ & $\begin{array}{l}3(6 \%) \\
47(94 \%)\end{array}$ & $<0.001 *$ \\
\hline Nasal Obstruction & $\begin{array}{l}\text { Yes } \\
\text { No }\end{array}$ & $\begin{array}{l}50(100 \%) \\
0(0 \%)\end{array}$ & $\begin{array}{l}8(16 \%) \\
42(84 \%)\end{array}$ & $<0.001 *$ \\
\hline Excessive Daytime Somnolence & $\begin{array}{l}\text { Yes } \\
\text { No }\end{array}$ & $\begin{array}{l}30(60 \%) \\
20(40)\end{array}$ & $\begin{array}{l}7(14 \%) \\
43(86 \%)\end{array}$ & $<0.001 *$ \\
\hline Nighttime Awakening & $\begin{array}{l}\text { Yes } \\
\text { No }\end{array}$ & $\begin{array}{l}40(80 \%) \\
10(20)\end{array}$ & $\begin{array}{l}5(10 \%) \\
45(90 \%)\end{array}$ & $<0.001 *$ \\
\hline
\end{tabular}

Non- significant $(p>0.05)$, significant $(p<0.05)$, -highly significant $(p<0.001)$.

Table 4: Comparison between study and control group regards to symptoms of OSA.

Also, we found $30 \%$ of the children in the study group had type B tympanogram in comparison to 6\% in the control group (table-5).

\begin{tabular}{|c|c|c|c|c|}
\hline \multirow{2}{*}{ Tympanogram } & & Case & control & \multirow{2}{*}{$\mathrm{P}$ value } \\
\hline & & $\mathrm{N}=50$ & $\mathrm{~N}=50$ & \\
\hline Preoperative & $\begin{array}{l}\text { Type A } \\
\text { Type B } \\
\text { Type C }\end{array}$ & $\begin{array}{l}16(32 \%) \\
15(50 \%) \\
19(38 \%)\end{array}$ & $\begin{array}{l}40(80 \%) \\
3(6 \%) \\
7(14 \%)\end{array}$ & $<0.001 *$ \\
\hline Postoperative & $\begin{array}{l}\text { Type A } \\
\text { Type B } \\
\text { Type C }\end{array}$ & $\begin{array}{l}43(86 \%) \\
4(8 \%) \\
3(6 \%)\end{array}$ & $\begin{array}{l}46(92 \%) \\
2(4 \%) \\
2(4 \%)\end{array}$ & 0.9 \\
\hline
\end{tabular}

Non- significant $(\mathrm{p}>0.05)$, significant $(\mathrm{p}<0.05)$, -highly significant $(\mathrm{p}<0.001)$.

Table 5: preoperative and postoperative Comparison between the study and control group regards to tympanogram.

The children in the study group had low IQ and low mental age in comparison to the control group (table-6).

\begin{tabular}{|c|c|c|c|c|}
\hline \multirow{2}{*}{\multicolumn{2}{|c|}{ Preoperative }} & Case & control & \multirow{2}{*}{$\mathrm{P}$ value } \\
\hline & & $\mathrm{N}=50$ & $\mathrm{~N}=50$ & \\
\hline IQ & $\begin{array}{l}\text { Range } \\
\text { Mean } \pm S D\end{array}$ & $\begin{array}{l}(70-91) \\
80.9 \pm 5.9\end{array}$ & $\begin{array}{l}(78-99) \\
89.1 \pm 4.3\end{array}$ & $<0.001 *$ \\
\hline Mental age (months) & $\begin{array}{l}\text { Range } \\
\text { Mean } \pm S D\end{array}$ & $\begin{array}{l}(54-132) \\
87.3 \pm 20.1\end{array}$ & $\begin{array}{l}(68-121) \\
95.9 \pm 22-3\end{array}$ & $<0.001 *$ \\
\hline
\end{tabular}

Non- significant $(\mathrm{p}>0.05)$, significant $(\mathrm{p}<0.05)$, -highly significant $(\mathrm{p}<0.001)$.

Table 6: preoperative and postoperative Comparison between the study and control group regards to the IQ and mental age.

The children in the study group had low score in all subtest of Illinois test of psycholinguistic abilities (table-7) and had low percentile of academic performance in comparison to the control group (table-8).

\begin{tabular}{|l|l|l|l|l|}
\hline \multirow{2}{*}{} & & Case & control & \multirow{2}{*}{ P value } \\
\cline { 3 - 4 } & & $\mathrm{N}=50$ & $\mathrm{~N}=50$ & \\
\hline \multirow{2}{*}{ PLA of AR } & Range & $\begin{array}{l}(40-106) \\
68.9 \pm 14.5\end{array}$ & $\begin{array}{l}(51-119) \\
79.8 \pm 16.5\end{array}$ & $<0.001^{*}$ \\
\hline \multirow{2}{*}{ PLA of VR } & Range $\pm S D$ & $(37-126)$ & $(53-131)$ & \multirow{2}{*}{$0.008^{*}$} \\
\hline \multirow{2}{*}{ PLA of ASM } & Mean $\pm S D$ & $81.5 \pm 23$ & $92.8 \pm 21.5$ & $<0.001^{*}$ \\
\hline \multirow{2}{*}{ PLA of VSM } & Reange $\pm S D$ & $(45-124)$ & $(55-136)$ & \\
& Range & $(37-123)$ & $87.3 \pm 18.8$ & $<0.001^{*}$ \\
\hline
\end{tabular}




\begin{tabular}{|c|c|c|c|c|}
\hline PLA of AA & $\begin{array}{l}\text { Range } \\
\text { Mean } \pm S D\end{array}$ & $\begin{array}{l}(43-124) \\
71.3 \pm 21\end{array}$ & $\begin{array}{l}(49-139) \\
86.5 \pm 11.4\end{array}$ & $<0.001 *$ \\
\hline PLA of VA & $\begin{array}{l}\text { Range } \\
\text { Mean } \pm S D\end{array}$ & $\begin{array}{l}(37-124) \\
76.9 \pm 18.6\end{array}$ & $\begin{array}{l}(48-139) \\
86.6 \pm 22.3\end{array}$ & $<0.001 *$ \\
\hline PLA of AC & $\begin{array}{l}\text { Range } \\
\text { Mean } \pm S D\end{array}$ & $\begin{array}{l}(36-126) \\
59.5 \pm 22.2\end{array}$ & $\begin{array}{l}(41-137) \\
71.5 \pm 31.6\end{array}$ & $<0.001 *$ \\
\hline PLA of VC & $\begin{array}{l}\text { Range } \\
\text { Mean } \pm S D\end{array}$ & $\begin{array}{l}(37-122) \\
64.2 \pm 18.4\end{array}$ & $\begin{array}{l}(49-138) \\
78.5 \pm 25.1\end{array}$ & $<0.001 *$ \\
\hline PLA of ME & $\begin{array}{l}\text { Range } \\
\text { Mean } \pm S D\end{array}$ & $\begin{array}{l}(36-123) \\
57.2 \pm 17.6\end{array}$ & $\begin{array}{l}(44-1382) \\
69.1 \pm 10.6\end{array}$ & $<0.032 *$ \\
\hline PLA of VE & $\begin{array}{l}\text { Range } \\
\text { Mean } \pm S D\end{array}$ & $\begin{array}{l}(39-94) \\
57.4 \pm 13\end{array}$ & $\begin{array}{l}(51-132) \\
71.8 \pm 19.3\end{array}$ & $<0.002 *$ \\
\hline PLA of GC & $\begin{array}{l}\text { Range } \\
\text { Mean } \pm S D\end{array}$ & $\begin{array}{l}(34-96) \\
55.7 \pm 13.7\end{array}$ & $\begin{array}{l}(55-138) \\
69.3 \pm 21.2\end{array}$ & $<0.001 *$ \\
\hline PLA of SB & $\begin{array}{l}\text { Range } \\
\text { Mean } \pm S D\end{array}$ & $\begin{array}{l}(38-97) \\
62.3 \pm 11.2\end{array}$ & $\begin{array}{l}(48-132) \\
77 \pm 14.5\end{array}$ & $<0.001 *$ \\
\hline Total PLA & $\begin{array}{l}\text { Range } \\
\text { Mean } \pm S D\end{array}$ & $\begin{array}{l}(50-98) \\
72.6 \pm 12.1\end{array}$ & $\begin{array}{l}(53-112) \\
93.1 \pm 12\end{array}$ & $<0.001 *$ \\
\hline
\end{tabular}

Non- significant $(\mathrm{p}>0.05)$, significant $(\mathrm{p}<0.05)$, -highly significant $(\mathrm{p}<0.001)$.

Table 7: preoperative Comparison between study and control state in the study group as regard the psycholinguistic age (PLA) of Illinois test.

\begin{tabular}{|l|c|c|c|c|}
\hline \multirow{2}{*}{ Academic performance } & Case & control & \multirow{2}{*}{ P value } \\
\cline { 3 - 4 } & Range & $\mathrm{N}=50$ & $\mathrm{~N}=50$ & \\
\hline \multirow{2}{*}{ Preoperative } & Mean $\pm S D$ & $(46-94)$ & $\begin{array}{c}(52-99) \\
86.3 \pm 77\end{array}$ & $<0.001 *$ \\
\hline
\end{tabular}

Non- significant $(\mathrm{p}>0.05)$, significant $(\mathrm{p}<0.05)$, -highly significant $(\mathrm{p}<0.001)$.

After eight months of adenotonsillectomy, the children in the study group had improvement in nasal tone of speech as $2 \%$ only has marked closed nasality (table-9) and most symptoms of OSA disappeared (table-10), only 8\% had type B tympanogram (table-11).

Table 8: preoperative Comparison between the study and control group regards to Academic performance.

\begin{tabular}{|c|c|c|c|}
\hline \multirow{2}{*}{ APA } & Preoperative & Postoperative & \multirow{2}{*}{ P value } \\
\cline { 2 - 3 } & $\mathrm{N}=50$ & $\mathrm{~N}=50$ & \\
\hline No Closed & $0(0 \%)$ & $22(44 \%)$ & \\
Mild & $0(0 \%)$ & $26(52 \%)$ & \multirow{2}{*}{$<0.001 *$} \\
Moderate & $32(64 \%)$ & $2(4 \%)$ & \\
Severe & $18(36 \%)$ & $0(0 \%)$ & \\
\hline
\end{tabular}

Non- significant $(\mathrm{p}>0.05)$, significant $(\mathrm{p}<0.05)$, -highly significant $(\mathrm{p}<0.001)$.

Table 9: Comparison between preoperative and postoperative state of the study group regards to APA.

\begin{tabular}{|c|c|c|c|c|}
\hline \multirow{2}{*}{\multicolumn{2}{|c|}{ Symptoms of OSA }} & Preoperative & Postoperative & \multirow{2}{*}{$P$ value } \\
\hline & & \multirow{2}{*}{$\begin{array}{l}\mathrm{N}=50 \\
50(100 \%) \\
0(0 \%)\end{array}$} & \multirow{2}{*}{$\begin{array}{l}\mathrm{N}=50 \\
5(10 \%) \\
45(90 \%)\end{array}$} & \\
\hline Snoring & $\begin{array}{l}\text { Yes } \\
\mathrm{No}\end{array}$ & & & $<0.001 *$ \\
\hline Mouth Breathing & $\begin{array}{l}\text { Yes } \\
\text { No }\end{array}$ & $\begin{array}{l}50(100 \%) \\
0(0 \%)\end{array}$ & $\begin{array}{l}3(6 \%) \\
47(94 \%)\end{array}$ & $<0.001^{*}$ \\
\hline Nasal Obstruction & $\begin{array}{l}\text { Yes } \\
\text { No }\end{array}$ & $\begin{array}{l}50(100 \%) \\
0(0 \%)\end{array}$ & $\begin{array}{l}8(16 \%) \\
42(84 \%)\end{array}$ & $<0.001 *$ \\
\hline Excessive Daytime Somnolence & $\begin{array}{l}\text { Yes } \\
\mathrm{No}\end{array}$ & $\begin{array}{l}30(60 \%) \\
20(40)\end{array}$ & $\begin{array}{l}7(14 \%) \\
43(86 \%)\end{array}$ & $<0.001^{*}$ \\
\hline Nighttime Awakening & $\begin{array}{l}\text { Yes } \\
\text { No }\end{array}$ & $\begin{array}{l}40(80 \%) \\
10(20)\end{array}$ & $\begin{array}{l}5(10 \%) \\
45(90 \%)\end{array}$ & $<0.001 *$ \\
\hline
\end{tabular}

Non- significant $(\mathrm{p}>0.05)$, significant $(\mathrm{p}<0.05)$, -highly significant $(\mathrm{p}<0.001)$. 


\begin{tabular}{|l|l|l|l|}
\hline \multirow{2}{*}{ Tympanogram } & Preoperative & Postoperative & \multirow{2}{*}{ P value } \\
\cline { 2 - 3 } & $\mathrm{N}=50$ & $\mathrm{~N}=50$ & \\
\hline Type $A$ & $16(32 \%)$ & $43(86 \%)$ & \multirow{2}{*}{$<0.001^{*}$} \\
Type $B$ & $15(50 \%)$ & $4(8 \%)$ & \\
Type $C$ & $19(38 \%)$ & $3(6 \%)$ & \\
\hline
\end{tabular}

Non- significant $(\mathrm{p}>0.05)$, significant $(\mathrm{p}<0.05)$, -highly significant $(\mathrm{p}<0.001)$.

\section{Table 11: Comparison between preoperative and postoperative state of the study group regards to tympanogram.}

The results revealed no statistically significant difference were obtained between the two groups regarding the mental age, IQ (table-12), as well as subtest of Illinois test of psycholinguistic abilities (table-13). Also, our data showed improvement in percentile of academic performances of the study group in comparison to the control group (table-14).

\begin{tabular}{|c|c|c|c|c|}
\hline \multirow{2}{*}{\multicolumn{2}{|c|}{ Postoperative }} & Case & control & \multirow{2}{*}{$\mathrm{P}$ value } \\
\hline & & $\mathrm{N}=50$ & $\mathrm{~N}=50$ & \\
\hline IQ & $\begin{array}{l}\text { Range } \\
\text { Mean } \pm S D\end{array}$ & $\begin{array}{l}(78-95) \\
84.1 \pm 5.6\end{array}$ & $\begin{array}{l}(78-99) \\
89.1 \pm 4.3\end{array}$ & 0.72 \\
\hline $\begin{array}{l}\text { Mental age } \\
\text { (Months) }\end{array}$ & $\begin{array}{l}\text { Range } \\
\text { Mean } \pm S D\end{array}$ & $\begin{array}{l}(61-133) \\
94.9 \pm 19.6\end{array}$ & $\begin{array}{l}68-121) \\
95.9 \pm 22-3\end{array}$ & 0.6 \\
\hline
\end{tabular}

Non- significant $(\mathrm{p}>0.05)$, significant $(\mathrm{p}<0.05)$, -highly significant $(\mathrm{p}<0.001)$.

Table 12: postoperative Comparison between the study and control group regards to the IQ and mental age.

\begin{tabular}{|c|c|c|c|c|}
\hline \multirow{2}{*}{\multicolumn{2}{|c|}{ psycholinguistic age (PLA) }} & \multirow{2}{*}{$\begin{array}{l}\text { Case } \\
\mathrm{N}=50\end{array}$} & \multirow{2}{*}{$\begin{array}{l}\text { control } \\
\mathrm{N}=50\end{array}$} & \multirow{2}{*}{$\mathrm{P}$ value } \\
\hline & & & & \\
\hline PLA of AR & $\begin{array}{l}\text { Range } \\
\text { Mean } \pm S D\end{array}$ & $\begin{array}{l}(47-118) \\
77.8 \pm 16.5\end{array}$ & $\begin{array}{l}(51-119) \\
79.8 \pm 16.5\end{array}$ & 0.4 \\
\hline PLA of VR & $\begin{array}{l}\text { Range } \\
\text { Mean } \pm S D\end{array}$ & $\begin{array}{l}(49-132) \\
90.8 \pm 22.5\end{array}$ & $\begin{array}{l}(53-131) \\
92.8 \pm 21.5\end{array}$ & 0.083 \\
\hline PLA of ASM & $\begin{array}{l}\text { Range } \\
\text { Mean } \pm S D\end{array}$ & $\begin{array}{l}(51-138) \\
85.3 \pm 20.6\end{array}$ & $\begin{array}{l}(55-136) \\
87.3 \pm 18.8\end{array}$ & 0.09 \\
\hline PLA of VSM & $\begin{array}{l}\text { Range } \\
\text { Mean } \pm S D\end{array}$ & $\begin{array}{l}(58-135) \\
75.1 \pm 18.5\end{array}$ & $\begin{array}{l}(60-138) \\
81.1 \pm 28.4\end{array}$ & 0.63 \\
\hline PLA of AA & $\begin{array}{l}\text { Range } \\
\text { Mean } \pm S D\end{array}$ & $\begin{array}{l}(48-138) \\
80.5 \pm 21.8\end{array}$ & $\begin{array}{l}(49-139) \\
86.5 \pm 11.4\end{array}$ & 0.43 \\
\hline PLA of VA & $\begin{array}{l}\text { Rangse } \\
\text { Mean } \pm S D\end{array}$ & $\begin{array}{l}(43-132) \\
82.6 \pm 19.6 \\
\end{array}$ & $\begin{array}{l}(48-139) \\
86.6 \pm 22.3\end{array}$ & 0.073 \\
\hline PLA of AC & $\begin{array}{l}\text { Range } \\
\text { Mean } \pm S D\end{array}$ & $\begin{array}{l}(43-138) \\
68.5 \pm 25.2\end{array}$ & $\begin{array}{l}(41-137) \\
71.5 \pm 31.6\end{array}$ & 0.5 \\
\hline PLA of VC & $\begin{array}{l}\text { Range } \\
\text { Mean } \pm S D\end{array}$ & $\begin{array}{l}(44-133) \\
71.5 \pm 20.9\end{array}$ & $\begin{array}{l}(49-138) \\
78.5 \pm 25.1\end{array}$ & 0.064 \\
\hline PLA of ME & $\begin{array}{l}\text { Range } \\
\text { Mean } \pm S D\end{array}$ & $\begin{array}{l}(36-132) \\
63.1 \pm 20.7\end{array}$ & $\begin{array}{l}(44-1382) \\
69.1 \pm 10.6\end{array}$ & 0.77 \\
\hline PLA of VE & $\begin{array}{l}\text { Range } \\
\text { Mean } \pm S D\end{array}$ & $\begin{array}{l}(40-124) \\
64.8 \pm 17.3\end{array}$ & $\begin{array}{l}(51-132) \\
71.8 \pm 19.3\end{array}$ & 0.8 \\
\hline PLA of GC & $\begin{array}{l}\text { Range } \\
\text { Mean } \pm S D\end{array}$ & $\begin{array}{l}(48-144) \\
66.3 \pm 21.2 \\
\end{array}$ & $\begin{array}{l}(55-138) \\
69.3 \pm 21.2\end{array}$ & 0.93 \\
\hline PLA of SB & $\begin{array}{l}\text { Range } \\
\text { Mean } \pm S D\end{array}$ & $\begin{array}{l}(46-127) \\
72 \pm 15.5\end{array}$ & $\begin{array}{l}(48-132) \\
77 \pm 14.5\end{array}$ & 0.66 \\
\hline Total PLA & $\begin{array}{l}\text { Range } \\
\text { Mean } \pm S D\end{array}$ & $\begin{array}{l}(50-98) \\
83.1 \pm 12\end{array}$ & $\begin{array}{l}(53-112) \\
93.1 \pm 12\end{array}$ & 0.8 \\
\hline
\end{tabular}

Non- significant $(\mathrm{p}>0.05)$, significant $(\mathrm{p}<0.05)$, -highly significant $(\mathrm{p}<0.001)$. 


\begin{tabular}{|l|l|l|l|l|}
\hline \multicolumn{2}{|c|}{ Academic performance } & Case & control & \multirow{2}{*}{ P value } \\
\cline { 2 - 4 } & $\mathrm{N}=50$ & $\mathrm{~N}=50$ & \\
\hline \multirow{2}{*}{ Postoperative } & Range & $(50-98)$ & $(52-99)$ & \multirow{2}{*}{0.8} \\
& Mean $\pm S D$ & $83.1 \pm 12$ & $86.3 \pm 77$ & \\
\hline
\end{tabular}

Non- significant $(\mathrm{p}>0.05)$, significant $(\mathrm{p}<0.05)$, -highly significant $(\mathrm{p}<0.001)$.

Table 14: postoperative Comparison between the study and control group regards to Academic performance.

Our results showed that, there were a Statistical significant differences between preoperative and postoperative results of the study group in the following parameters, the PLA of AR (auditory reception), VR (visual reception), auditory sequential memory, VE (Visual Expression), ME (Mental Expression) as $\mathrm{p}<0.05$ ). Also, Statistically significant differences were obtained between the preoperative and postoperative of the study group concerning the PLA of VSM (Visual sequential memory), AA (Auditory association) VA (Visual association), AC (Auditory closure),
VC (Visual closure), GC (Grammatical closure) and SB (Sound Blending) as $\mathrm{p}<0.001)$. The current study pointed out that a statistically significant differences were obtained between the preoperative and postoperative results of the study group in the the Total PLA. $(\mathrm{p}<0.001)$. The mean Total PLA in the preoperative examination of the study group were $72.6 \pm 12.1$ with a range (50-98) months, and the mean Total PLA in the postoperative data of the study group were $83.1 \pm 12$ with a range (5098) months (Table-15).

\begin{tabular}{|c|c|c|c|c|}
\hline \multirow{2}{*}{\multicolumn{2}{|c|}{ psycholinguistic age (PLA) }} & Preoperative & Postoperative & \multirow{3}{*}{$\begin{array}{l}\text { P value } \\
<0.001\end{array}$} \\
\hline & & \multirow{2}{*}{$\begin{array}{l}N=50 \\
(40-106) \\
68.9 \pm 14.5\end{array}$} & \multirow{2}{*}{$\begin{array}{l}N=50 \\
(47-118) \\
77.8 \pm 16.5\end{array}$} & \\
\hline PLA of AR & $\begin{array}{l}\text { Range } \\
\text { Mean } \pm S D\end{array}$ & & & \\
\hline PLA of VR & $\begin{array}{l}\text { Range } \\
\text { Mean } \pm S D\end{array}$ & $\begin{array}{l}(37-126) \\
81.5 \pm 23\end{array}$ & $\begin{array}{l}(49-132) \\
90.8 \pm 22.5\end{array}$ & $0.008 *$ \\
\hline PLA of ASM & $\begin{array}{l}\text { Range } \\
\text { Mean } \pm S D\end{array}$ & $\begin{array}{l}(45-124) \\
72 \pm 19.8\end{array}$ & $\begin{array}{l}(51-138) \\
85.3 \pm 20.6\end{array}$ & $<0.001 *$ \\
\hline PLA of VSM & $\begin{array}{l}\text { Range } \\
\text { Mean } \pm S D\end{array}$ & $\begin{array}{l}(37-123) \\
67.9 \pm 16.8\end{array}$ & $\begin{array}{l}(58-135) \\
75.1 \pm 18.5\end{array}$ & $<0.001 *$ \\
\hline PLA of AA & $\begin{array}{l}\text { Range } \\
\text { Mean } \pm S D\end{array}$ & $\begin{array}{l}(43-124) \\
71.3 \pm 21\end{array}$ & $\begin{array}{l}(48-138) \\
80.5 \pm 21.8\end{array}$ & $<0.001 *$ \\
\hline PLA of VA & $\begin{array}{l}\text { Range } \\
\text { Mean } \pm S D\end{array}$ & $\begin{array}{l}(37-124) \\
76.9 \pm 18.6\end{array}$ & $\begin{array}{l}(43-132) \\
82.6 \pm 19.6\end{array}$ & $<0.001 *$ \\
\hline PLA of AC & $\begin{array}{l}\text { Range } \\
\text { Mean } \pm S D\end{array}$ & $\begin{array}{l}(36-126) \\
59.5 \pm 22.2\end{array}$ & $\begin{array}{l}(43-138) \\
68.5 \pm 25.2\end{array}$ & $<0.001 *$ \\
\hline PLA of VC & $\begin{array}{l}\text { Range } \\
\text { Mean } \pm S D\end{array}$ & $\begin{array}{l}(37-122) \\
64.2 \pm 18.4\end{array}$ & $\begin{array}{l}(44-133) \\
71.5 \pm 20.9\end{array}$ & $<0.001^{*}$ \\
\hline PLA of ME & $\begin{array}{l}\text { Range } \\
\text { Mean } \pm S D\end{array}$ & $\begin{array}{l}(36-123) \\
57.2 \pm 17.6\end{array}$ & $\begin{array}{l}(36-132) \\
63.1 \pm 20.7\end{array}$ & $<0.032 *$ \\
\hline PLA of VE & $\begin{array}{l}\text { Range } \\
\text { Mean } \pm S D\end{array}$ & $\begin{array}{l}(39-94) \\
57.4 \pm 13\end{array}$ & $\begin{array}{l}(40-124) \\
64.8 \pm 17.3\end{array}$ & $<0.002 *$ \\
\hline PLA of GC & $\begin{array}{l}\text { Range } \\
\text { Mean } \pm S D\end{array}$ & $\begin{array}{l}(34-96) \\
55.7 \pm 13.7\end{array}$ & $\begin{array}{l}(48-144) \\
66.3 \pm 21.2 \\
\end{array}$ & $<0.001 *$ \\
\hline PLA of SB & $\begin{array}{l}\text { Range } \\
\text { Mean } \pm S D\end{array}$ & $\begin{array}{l}(38-97) \\
62.3 \pm 11.2\end{array}$ & $\begin{array}{l}(46-127) \\
72 \pm 15.5\end{array}$ & $<0.001 *$ \\
\hline Total PLA & $\begin{array}{l}\text { Range } \\
\text { Mean } \pm S D\end{array}$ & $\begin{array}{l}(50-98) \\
72.6 \pm 12.1\end{array}$ & $\begin{array}{l}(50-98) \\
83.1 \pm 12\end{array}$ & $<0.001 *$ \\
\hline
\end{tabular}

Non- significant $(\mathrm{p}>0.05)$, significant $(\mathrm{p}<0.05)$, -highly significant $(\mathrm{p}<0.001)$.

Table 15: Comparison between preoperative and postoperative state in the study group as regard the psycholinguistic age (PLA) of Illinois test.

A Statistical significant differences were obtained between the preoperative and postoperative results of the study group as regard Academic performance. $(\mathrm{p}<0.05)$. The mean Academic performance in the preoperative examination of the study group were $66.6 \pm 12.1$ with a range (46-70) percentage, and the mean Total row score in the postoperative examination of the study group were $83.1 \pm 12$ with a range (60-98) percentage (Table-16). 


\begin{tabular}{|l|l|l|l|}
\hline \multirow{2}{*}{ Academic performance } & Preoperative & Postoperative & \multirow{2}{*}{ P value } \\
\cline { 2 - 4 } & $\mathrm{N}=50$ & $\mathrm{~N}=50$ & \\
\hline Range & $(46-94)$ & $(50-98)$ & $<0.001 *$ \\
Mean $\pm S D$ & $66.6 \pm 12.1$ & $83.1 \pm 12$ & \\
\hline
\end{tabular}

Non- significant $(p>0.05)$, significant $(p<0.05)$, -highly significant $(p<0.001)$.

Table 16: Comparison between preoperative and postoperative state as regard to Academic performance in the study group.

\section{Discussions}

In children, OSA is a disorder characterized by repeated and/or prolonged airway obstructions during sleep, in the form of apneas and hypopneas throughout the night [1]. OSAS exhibits specific cognitive dysfunctions that include deficits in memory, problem-solving, and behavioral functioning [14-15]. This is problematic, as SDB with AHI < 1 can still impact children's academic performance and behavior [16]. Friedman et al. [17] reported that young children who snore frequently and loudly during sleep are at greater risk for poor academic performance in later years, even well after snoring has resolved, suggests that the neurocognitive deficits may be only partially reversible after treatment. AT results in significant improvement in clinical as well as polysomnography parameters. Improvement in learning and behavior has been reported following the treatment of OSAS in children. The purpose of our present study was to evaluate the effect of AT on the academic and cognitive achievement of children with OSA so proper management of those children can be established. The study group included 50 children well diagnosed as OSA due to adenotonsillar hypertrophy confirmed by polysomnography compared to another control group of 50 children without symptoms of OSA.

The study group reported nighttime symptoms, including mouth breathing, apneic episodes, frequent awakenings, and restlessness. Children also had enuresis, behavior problems, deficient attention span, and daytime somnolence. The study group improved most of OSAS symptoms post AT, and this attributed to the removal of the upper airway obstruction. $60 \%$ of patients in our study had a severe degree of hyponasal speech, and $40 \%$ had a moderate degree of hyponasal speech pre-AT, but 8 months post AT only $4 \%$ of the patients had moderate hyponasal speech, and $12 \%$ had a mild degree of hyponasal speech. This improvement of the auditory perceptual assessment can be attributed to the removal of the obstructive effect of adenoid on the choana, i.e., get rid of the anatomic and physiological factors that constrict the space of nasopharynx and compromising the soft tissues surrounding the pharynx that predispose to upper airway collapse and change in normal resonance of the vocal tract.

Cognition is a mental act that includes perception, reasoning, awareness, and intuition. Executive function encompasses the mental processes that enable children to plan, focus, remember instructions, and juggle multiple tasks successfully.

Psycholinguistics evaluations were carried out before and after AT for our patients revealed a significant difference between children in the study and the control group. The mean IQ of children in our study was increasing from $80.9 \pm 5.9$ (pre AT) to $84.1 \pm 5.6$ (post AT). Our result revealed a negative significant correlation between AHI and IQ, of the study group ( $\mathrm{p}<0.001)$. This improvement of IQ in our patients with OSA can be attributed to the improvement of the upper airway obstruction during sleep, disappearance of intermittent hypoxemia, improvement of ventilation, and enough hours of a deep sleep. Eight months post AT considered enough duration to raise intellectual functions, so improvement in IQ was detected. These results match most of the published data about the improvement of IQ after AT in children with OSA. As Friedman et al., [17] conducted a prospective study to evaluate neurocognitive functions of children with OSAS, before and after AT, he found that six to ten months after adenotonsillectomy, the children with OSAS demonstrated significant improvement in sleep characteristics, as well as in daytime behavior, and their neurocognitive performance improved considerably in most tests conducted. However, on the other side Abd-Allatif et al. [18] in their study stated that the intelligent quotient (IQ) as an indicative parameter of neurocognitive function in children with adenoid hypertrophy not improved with adenoidectomy and there is no relation between adenoid hypertrophy and IQ. Taylor et al. [19] stated that significant improvement in the NESPY Auditory attention and response set of children with OSA seven months after early AT Children with primary snoring, as well as those with OSA, are at higher risk for deficits in attention compared to control children when measured on parental report scales, and that such deficits are substantially improved following treatment with AT [9].

The mean PLA of Auditory association, visual association, auditory closure, visual closure, and manual expression, were raised 8 months after AT. This wide improvement in neurocognitive functions was attributed to the correction of the obstructive symptoms, improvement of sleep disruption, intermittent hypoxemia and inattention after AT, and in turn the improvement of the neurocognitive function as well as the intellectual functions demonstrated from the results of the IQ. We think that normal language development in children is such an important factor in acquisition of reading and writing skills. Most children with OSA had a history of delayed language development during their early life. Phonetic sounds are acquired between ages 3-7 years, which is also the peak age for hypertrophy of the tonsils and childhood OSA. Our results matched to Correa et al., [20] who stated that the late diagnosis and treatment of OSA is associated with a delay in verbal skill acquisition.

The study that done by Marcus et al, [10] it evaluate the difference between adenotonsillectomy and wait and watch policy for management of OSAS, the study was randomized, multicenter and controlled trial. It measures cognitive, Polysomnography and behavioral patterns, At baseline and seven months after AT, they found that As compared with a strategy of watchful waiting, surgical treatment for OSAS in school-age children did not significantly improve executive function or attention when measured by neuropsychological testing but did reduce symptoms and improve secondary outcomes of behavior, quality of life, and polysomnographic findings, thus providing evidence of beneficial effects of early AT.

we found that children with OSA due to adenotonsillar hypertrophy had poor academic achievement owing to obstructive symptoms of upper respiratory tract but eight months post adenotonsillectomy there were improvement of 12 points of Illinois test in the Mental Processing and increasing in the academic performance also Parents of our children noticed that their children became more attentive and slept better without snoring, less impulsive and hyperactive.

The diagnosis and treatment of OSA should be emphasized, not only because of the possible implications for oral language, as demonstrated in the reviewed studies, which tend to worsen as the chronological age increases but also for the benefits in neurocognitive performance and quality of life. Weber et al. [21] stated that OSA children show a higher frequency of disorders of receptive and expressive language skills, mostly under diagnosed and thus not treated. A more multi-disciplinary 
investigation of OSA children is needed to foster the evidence. In current study, there were increase in the score of PLA of VE (Verbal Expression) and grammatical closure. This improvement can be attributed to children with OSA due to adenotonsillar hypertrophy and Eustachian tube dysfunction, positive middle ear pressure, middle ear fluid accumulation, conductive hearing loss, all these causes impaired language acquisition, causing impairment in language development (semantic, syntactic, phonological and pragmatic development).

Ikedia et al [22] reported that to assess whether tonsillectomy and AT surgeries would bring benefits as far as school performance is concerned in children with OSA, psychological evaluations were carried out before and after the surgery. Gozal \& Pope [22] investigated 300 children with the worst grades in a school, $18.1 \%$ of them had gas-exchange $(\mathrm{O} 2$ and $\mathrm{CO} 2$ ) disorders during sleep. Of those with obstructive sleep disorders, half were submitted to adenoidectomy. They noticed that the group submitted to adenoidectomy had a significant increase in school performance. The other half, without disorders, kept their mean scores. AT is effective in treating breathing problems in most children with OSAS and also appears to help improve their academic and intellectual performance and general behavior [17].

In the current study, eight months post AT, the mean Academic performance was increasing from (post AT) in comparison to the control group. This improvement was demonstrated from increasing in the scores of the children' school subjects also from the results of the tests carried out (Stanford Binet Intelligence Scale and Illinois tests). Parents / guardian of the study group noticed that their children became more attentive, no snoring, sleep better, less hyperactive and less impulsive All these changes will Benefit the entire family. The improvement in the previous parameters attributed to correction of frequent arousals during sleep, intermittent nocturnal hypoxia, anxiety, impulsivity, deficits in emotional regulation, alertness, inattentive behaviors, And attention to tasks after removal of Adenoid and tonsils that obstruct upper airway. This correction leads to improvement in the memory and IQ, resulting in an increase in academic performance. The improvement of language expression,, which was impaired in the Illinois subsets, after surgical correction of OSA, This correction lead to increase in the mean age of the verbal expression, grammatical closure and sound blending subtests of Illinois test After eight months post AT than pre AT.

\section{Conclusion}

Language is a very important factor for good academic achievement so this important finding can help in the prediction of poor academic achievement or learning disabilities through appreciation of language impairment; also we raise attention to the importance of early detection or screening for language impairment in children in the hope of preventing possible late severe learning disabilities. This study (up to our knowledge) is the first study to use Illinois test to determine specific strengths and weaknesses among language abilities, for early identification of children at-risk for school failure. An improvement of 12 points of Illinois' test in the Mental Processing following AT is a very substantial improvement. This change enables the children to reach their original abilities and fulfill their cognitive potential. Cognitive factors such as memory, attention, intellectual and school performance improved significantly after AT. Visual and auditory short-term memory, as well as the ability to solve problems and to think analytically improved. These findings suggest that OSAS is a reversible disruptive factor to the neurocognitive function and school performance of children, at least in the short term (8 months after therapy).

Conflict of interest: None

Funding: None
Deceleration: The study was approved by the research ethics committee of the Faculty of Medicine and in agreement with Helsinki declaration. Informed signed consent is taken from the parents/ guardian of the children.

\section{References:}

1. American Academy of Pediatrics, Subcommittee on Obstructive Sleep Apnea Syndrome. Clinical practice guideline: diagnosis and management of childhood obstructive sleep apnea syndrome. Pediatrics 2002; 109: 704-711.

2. Brietzke SE, Gallagher D., The effectiveness of tonsillectomy and adenoidectomy in the treatment of pediatric obstructive sleep apnea/hypopnea syndrome. A meta-analysis. Otolaryngology Head \& Neck Surgery 2006; 134(6): 979-984.

3. Tauman R, Gozal D., Obesity and obstructive sleep apnea in children. Pediatric Respiratory Reviews 2006; 7(4):247-259.

4. Ong TH., Obstructive sleep apnea syndrome: clinical features in children vs. adults. Medicine \& Health, Rhode Island 2006; 89(3): 104-106.

5. Bhattacharyya N, Lin HW., Changes and consistencies in the epidemiology of pediatric adenotonsillar surgery 1996-2006. Otolaryngol Head Neck Surg 2010; 143:680-684.

6. O'Brien L, Gozal D., Consequences of Sleep Apnea Syndrome. In S.D. Sheldon, R; Kryger, MH; Ferber, R (Ed.), Principles and Practice of Pediatric Sleep Medicine, 2006.p.212-218.

7. Bucks RS, Olaithe M, Eastwood P., Neurocognitive function in obstructive sleep apnea: A meta-review, Official Journal of Asian Pacific Society of Respiration 2012; 18: 61-70.

8. O'Brien LM, Mervis CB, Holbrook CR, et al., Neurobehavioral implications of habitual snoring in children. Pediatrics 2004; 114:44-49.

9. Gozal D. Obstructive Sleep Apnea in Children: Implications for the Developing Central Nervous System, Semin Pediatr Neurol 2008; 15(2): 100-106.

10. Marcus CL, Reneé H, Rosen CL, et al., A Randomized Trial of Adenotonsillectomy for Childhood Sleep Apnea. N Engl J Med. 2013 20; 368(25): 2366-2376.

11. Kotby, MN (1986) Voice disorders: recent diagnostic advances. Egypt. J. Otolaryngol.; 3(1): 69.

12. Berry RB, Budhiraja R, Gottlieb DJ, et al., Rules for scoring respiratory events in sleep:. J Clin Sleep Med 2012; 8: 597-519.

13. Gary CL., Orthogonal Higher Order Factor Structure of the Stanford-Binet Intelligence Scales- Fifth Edition for Children and Adole-scents . School Psychology Quarterly 2008; 23: 533-541.

14. Adel Aziz A, Shaheen EA, Osman DM., Arabic psycholinguistic screening tool: A preliminary study. The Egyptian Journal of Otorhinolaryngology 2012; $28: 64-73$.

15. Wang Y, WANG JJ, ZHA, SM., Changes of Serum Brain-derived M-Q Neurotrophic Factor in Children with Obstructive Sleep Apnea-Hypopnea Syndrome following Adenotonsillectomy. The Journal of International Medical Research 2010; 38: 1942 -1951.

16. Rosen CL, Storfer-Isser A, and Taylor HG, et al. Increased behavioral morbidity in school-aged children with sleepdisordered breathing. Pediatrics 2004; 114: 1640-1648.

17. Friedman BC, Amitai AH, Kozminsky E, Adenotonsillectomy improves neurocognitive function in children with obstructive sleep apnea syndrome. Sleep J 2003; 26: 999 - 1005.

18. Abd-Allatif M, Ibrahim H, Yehia S, et al., The Effect of A adenoid Hypertrophy On Intelligence Quotient At Preschool Age. AAMJ 2014; 12:344-360.

19. Taylor HG, Bowen SR, Beebe DA et al. Cognitive Effects of Adenotonsillectomy for Obstructive Sleep Apnea. Pediatrics 2018; 38:66-98. 
20. Corrêa CC, CavalheiroM G, Maximino LP., Obstructive sleep apnea and oral language disorders, Braz. j. otorhinolaryngol. 2017; 83:212-226.

21. Weber S, Correa C, Abramides DV: Oral language performance in children with obstructive sleep apnea. European Respiratory Journal 2016; 48: 43-54.
22. Ikeda FH, de Campos Horta HP, et al., Intellectual and school performance evaluation of children submitted to tonsillectomy and adenotonsillectomy before and after surgery. Braz J Otorhinolaryngol 2012;78(4):17-23.

23. Gozal D, Pope D: Snoring during early childhood and academic performance at ages 13-14 years. Pediatrics 2001; 107: 13941399.
This work is licensed under Creative Commons Attribution 4.0 License

\section{To Submit Your Article Click Here: Submit Manuscript}

DOI: $10.31579 / 2578-8868 / 147$
Ready to submit your research? Choose Auctores and benefit from:

fast, convenient online submission

* rigorous peer review by experienced research in your field

* rapid publication on acceptance

* authors retain copyrights

* unique DOI for all articles

* immediate, unrestricted online access

At Auctores, research is always in progress.

Learn more www.auctoresonline.org/journals/neuroscience-andneurological-surgery 